Voix et Images

voixetimages

\title{
Mourir au théâtre
}

\section{Lucie Robert}

Volume 27, numéro 1 (79), automne 2001

\section{Fernand Dumont}

URI : https://id.erudit.org/iderudit/201593ar

DOI : https://doi.org/10.7202/201593ar

Aller au sommaire du numéro

\section{Éditeur(s)}

Université du Québec à Montréal

\section{ISSN}

0318-9201 (imprimé)

1705-933X (numérique)

Découvrir la revue

Citer cet article

Robert, L. (2001). Mourir au théâtre. Voix et Images, 27(1), 151-158.

https://doi.org/10.7202/201593ar

Ce document est protégé par la loi sur le droit d'auteur. L'utilisation des services d'Érudit (y compris la reproduction) est assujettie à sa politique d'utilisation que vous pouvez consulter en ligne.

https://apropos.erudit.org/fr/usagers/politique-dutilisation/
Cet article est diffusé et préservé par Érudit.

Érudit est un consortium interuniversitaire sans but lucratif composé de l’Université de Montréal, l'Université Laval et l'Université du Québec à Montréal. Il a pour mission la promotion et la valorisation de la recherche. https://www.erudit.org/fr/ 


\section{Dramaturgie}

\section{Mourir au théâtre}

\section{Lucie Robert, Université du Québec à Montréal}

La mort fascine le théâtre et, parmi les lieux communs les plus tenaces, figure l'idée que le rêve de tout comédien est de mourir sur scène, comme Molière. Mais si la mort fascine au théâtre, c'est d'abord qu'elle en représente l'interdit. Mourir est un geste irréversible qui n'arrive qu'une fois, car l'état de mort dure. Or le théâtre est tout le con- traire de cette durée: il est réversible, répétitif et éphémère. À la fin de la représentation, l'acteur abandonne le personnage et redevient lui-même alors que ce retour de l'état de jeu vers la réalité dévoile le caractère fictif des événements représentés. Si la mort fascine tant le théâtre, c'est aussi parce.qu'elle représente la forme ultime de toute conclusion. À 
rebours, la vie devient cohérente et interprétable, comme une trajectoire qui aurait été tracée d'avancé et suivie attentivement. Toutes ces considérations ne concernent bien sûr qu'une mort esthétisée, qui entretient une vague confusion avec la chose réelle. En ce sens, la mort au théâtre est toujours "la mort des autres ${ }^{1}$ " qui, chaque fois, permet de flirter avec la sienne propre.

Mourir au théâtre n'est toutefois pas sans offrir des modèles, comme le rappelle Robert Lalonde dans l'avant-propos de sa première pièce, Monsieur Bovary ou mourir au théâ$t^{2}{ }^{2}$ : "J'ai alors imaginé que Gustave revoyait tout son monde, ses personnages, ses amis, ses ennemis, tous réunis autour de son grand corps fatigué - le jour même de sa mort. Une mort semblable à celle de Molière, dans le film d'Ariane Mnouchkine, montant, à bout de souffle, un interminable escalier, menant de la scène au ciel." (p. 9) La figure de Molière est tenace et y superposer celle de Flaubert marque les choix esthétiques fondamentaux de l'auteur, connu d'abord comme comédien et romancier: "J'aime Gustave, depuis toujours." (p. 7) La pièce, mise en scène par Lorraine Pintal, et créée le 18 janvier 2001 au Théâtre du Nouveau Monde, en co-production avec le Trident et le Théâtre Français du Centre National des Arts, aurait été écrite à la suite d'un défi lancé par des collègues comédiens: "S'il est si "hénaurmément" formidable que ça, ton Flaubert, pourquoi tu n'en fais pas un personnage de théâtre?" (p. 8) Mettre en scène un romancier qui, toute sa carrière, a "vomi le théâtre ", faute d'y être parvenu, était en effet une gageure.
Structurée en deux parties et dixneuf tableaux, la pièce s'ouvre sur un personnage qui a déjà entrepris sa mort et elle se termine au moment du passage final. Flaubert apparaît "comme une momie dans sa longue éternité ", selon le titre du premier tableau, et il règle ses derniers comptes avec les êtres réels ou fictifs qui l'habitent. Emma, Salammbô, Bouvard et Pécuchet rencontrent leurs modèles: Louise, Caroline, les frères Goncourt, mais aussi la mégère apprivoisée de Shakespeare ou l'une ou l'autre des filles du roi Lear. George Sand paraît dans le rôle de la Grande Faucheuse; Maupassant agit comme meneur de jeu et comme passeur; Tourgueniev est appelé à témoigner de la valeur de l'œuvre. Louis Bouilhet, l'obscur auteur dramatique, rappelle l'expérience du théâtre, à travers la figure du Cyrano de Rostand: "C'est notre histoire, Gustave, et puis voilà tout." (p. 70) Outre Shakespeare et Rostand, Molière, Racine, Hugo, Musset et Balzac sont encore convoqués comme modèles ou comme repoussoirs.

Certes, Lalonde connaît Flaubert : il a visiblement lu l'ensemble des romans et la correspondance, ce qui lui permet de créer un personnage complexe avec beaucoup de raffinement sans pour autant proposer d'interprétation nouvelle. Les tableaux se succèdent comme autant de taches sur une toile contemporaine: saisir l'effet d'ensemble exige toutefois une perspective particulière, un point de fuite éloigné, situé au-delà du texte luimême. Il nous faut déjà connaître Flaubert pour saisir le portrait qu'en trace Lalonde. Aussi l'écrivain n'apparaît-il jamais comme un véritable personnage de théâtre: il reste 
comme le point de fuite intra-textuel à partir duquel les autres personnages se développent. Hommage à Gustave, donc à l'artiste et à l'homme, la pièce est loin de l'idée que Flaubert lui-même se faisait du théâtre: "Le théâtre, c'est fait pour provoquer, non? Pour soulever l'indignation, désengager le foie d'un tropplein de bile, non?" (p. 65)

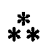

L'aburissant vertige de M. Maelström ${ }^{3}$ de Claude Paiement reprend l'idée d'explorer le moment de la mort, mais dans un registre tout autre. La pièce n'est pas neuve, ayant été créée en novembre 1997, à l'Espace La Veillée par le théâtre Harpagon (Ô Molière!), dans une mise en scène de l'auteur. Elle est, cependant, éditée dans une toute nouvelle collection dirigée par l'École nationale de l'Humour chez Lanctôt, collection inaugurée l'an dernier par la réédition de l'œuvre intégrale d'Yvon Deschamps. L'objectif des éditeurs est de "rattraper " les textes comiques, peu nombreux et peu traditionnels dans la dramaturgie québécoise, qui, négligés par les grands éditeurs de théâtre, tombent généralement dans l'oubli.

Pièce de facture classique, $L^{\prime} a$ burissant vertige de M. Maelström met en scène les derniers moments de la vieillesse, cet âge où le corps ne répond plus et où, dit-on, la tête n'est pas toujours certaine. Il s'agit d'un comique de caractère alors que Maelström se refuse à ouvrir la porte à un huissier dont les coups ponctuent le rythme de la pièce. Autour de lui, gravitent un secrétaire un peu bouffon qui n'a pas été payé depuis des lustres et qui agit comme meneur de jeu; une fille dont on ne sait pas bien si elle vole son père ou si elle le protège, et divers personnages empruntés à la tradition du genre: la prostituée (qui confirme l'échec du corps); le médecin (qu'on renvoie, faute de remède); l'avocat (qu'on renvoie aussi, faute de coupable à poursuivre); le charlatan (qui se trouve être celui qui révèle la vérité). La pièce est composée de répliques courtes qui se succèdent sur le mode de la stichomythie. Elle repose sur une succession de quiproquos qui ne seront pas tous résolus et permet de nombreux jeux de scène. Mais le vieux est faible; le docteur, l'avocat, le secrétaire et le charlatan sont ordinaires alors que la fille est plutôt insignifiante. Dans cette pièce malgré tout structurée efficacement, le propos reste mince. C'est la résistance de Maelström devant l'inéluctable qui est tournée en dérision: depuis la construction d'un système à manivelle permettant d'accomplir l'acte sexuel quand le corps s'y refuse jusqu'à la quête du remède miracle, malheureusement inexistant. Il n'y a ici aucun élément qui permette soit de tracer le bilan d'une vie, soit de mesurer le tragique de la fin ou même d'en saisir l'absurdité, ce que suggèrerait pourtant le sous-titre $\mathrm{Va}$ riations jazzées sur un thème ionesquien. Loin de ce modèle, le comique est réduit à l'humour, aux jeux de mots et aux jeux de scène, à la caricature et au ridicule.

\section{**}

Plus intéressante est la situation initiale que crée Gilles-Philippe Pelletier: "La rage peut éclater en quinze 
jours ou s'étendre sur plusieurs années [...] On connaît des cas où elle a dépassé cinq, dix, vingt et même trente ans, sans erreur possible..." (p. 71) Dans la gueule d'un chien. Pièce en un acte ${ }^{4}$ a été mise en lecture par l'auteur et présentée au Studio d'essai Claude-Gauvreau (UQÀM), en octobre 1998. Il ne s'agit pas de la première pièce de l'auteur, qui en a écrit une vingtaine d'autres parmi lesquelles six ont été jouées, mais il s'agit de la première à faire l'objet d'une publication. JeanBaptiste a autrefois été mordu par un chien enragé. Il est le dernier survivant de ce village dont les habitants sont tous morts des conséquences directes ou indirectes de la rage. Sentant la maladie éclater, quelque quarante ans plus tard, il écrit la pièce, comme pour témoigner. L'action se passe pendant l'été 1998, dans un village fantôme nommé SainteNégativité, situé sur les battures du Bas Saint-Laurent. Au fond, en décor, l'ombre de la "Cacapitale" d'État. Le personnage principal de la pièce de Jean-Baptiste est un chien, Météore: "depuis des lunes, ce chien erre sur les rives du fleuve à la recherche d'un maitre" (p. 13). Cependant, précise l'auteur, le rôle doit être joué par un acteur qu'on n'affabulera pas d'un museau, car "Il est plus facile de diriger le jappement d'un acteur que de domestiquer les sentiments d'un chien." (prologue) Le chien a la rage et il mord chacun de ses maîtres, avec les résultats qu'on suppose.

Dans le village, ne résident plus que des fantômes, spectres d'une époque sans âge, des riverains, qui ne demandent qu'à s'embarquer sur le traversier de l'île Blanche, "L'endroit où vont les humains après leur mort " (p. 26). Tous ces personnages ont déjà eu une vie réelle, un drame personnel. Ils ont tous été mordus et la mort les a saisis dans un ordre indéfini. Rachel, la petite fille, a eu le temps d'étudier la musique, d'avoir un fils et de refaire sa vie. Antoine, ce fils, n'a eu qu'une enfance avant de se piquer avec la seringue destinée à mettre fin aux jours de Météore. Marguerite, l'institutrice a trouvé le chien attaché et elle l'a emmené à l'école pour le montrer à ses élèves. Le chien a mordu un enfant. L'enfant se nommait JeanBaptiste: "Jean-Baptiste! c'est mon nom! / Mon nom est tatoué sur le crâne des enfants de nos ennemis! Et toi le chien, comment t'appellestu? Hamlet ! [...] Pourquoi tiens-tu un crâne d'enfant dans ta gueule?" (p. 70)

La référence à Hamlet engendre une autre lecture de la pièce, où la rage devient la métaphore d'une quête plus fondamentale: loin d'être linéaire, la pièce crée des espaces et des trames parallèles pour parler d'Anne-Marie, de Rachel et d'Antoine qui, comme Jean-Baptiste, ressentent de manière douloureuse l'absence du père. Charles, Philippe, le violeur remontent comme autant de souvenirs cruels dans la mémoire de JeanBaptiste. Quête du père, mais aussi quête du pays, écrit l'auteur, mais de quel pays? Car le seul pays désiré par les riverains, c'est le fleuve et son île Blanche. Lîle fantôme.

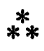

Comme plusieurs jeunes auteurs, Serge Boucher emprunte à Tchékhov l'idée d'une dramaturgie du quoti- 
dien, sans action dramatique réelle, qui tente de mettre en scène la vie comme une durée, comme une durée lourde, un présent sans fin. Il inverse toutefois le principe en insérant dans cette durée, relativement euphorique, un élément perturbateur, un instantané, qui vient bouleverser la vie quotidienne. Il en résulte une dramaturgie sans action, des textes sans véritable début ni véritable fin.

24 poses $^{5}$, créée au Théâtre d'Aujourd'hui le 3 novembre 1999 , dans une mise en scène de RenéRichard Cyr, réfère bien sûr à la photographie (la pièce est d'ailleurs sous-titrée Portraits), et elle est construite comme un discours d'escorte, un commentaire auquel se livreraient les membres d'une famille réunie autour de l'album de photographies, souvenirs du jour anniversaire du fils aîné, Richard. L'action se passe dans la cour arrière du plus banal bungalow de banlieue. Nous aurons droit aux remarques sur le temps qu'il fait, la qualité de la nouvelle tondeuse à gazon, les soldes du supermarché. Sans être dysfonctionnelle, la famille craque de partout. Le père est un alcoolique repenti, devenu propriétaire d'un dépanneur quelque part dans les Bois-Francs. Son vieux cœur est malade, il voudrait bien prendre sa retraite, mais ne comprend pas grand-chose au monde moderne. La mère rêve de gagner à la loterie pour effacer un passé assez pénible et s'offrir quelques petits plaisirs. La fille, trop gaie, trop volubile, s'est dotée d'un amant un peu trouble, un peu menteur, un peu voleur. Le fils cadet, l'inévitable professeur de littérature, écrivain manqué, traverse visiblement une crise dont on ne connaîtra jamais le véritable sens. L'oncle en chômage, trop vieux pour espérer trouver un nouvel emploi, est trop gentil et trop reconnaissant envers sa sœur et son neveu qui le font vivre. Seuls, les deux hôtes, de condition très modeste, un peu naifs et un peu perdus, paraissent conserver quelque équilibre. Entre les inévitables caisses de vingt-quatre, la sauce à spaghetti et la tondeuse à gazon, les personnages entretiennent une conversation artificielle dont la seule fonction est de maintenir la cohésion familiale. Le suicide de l'oncle mettra une fin à cette journée comme tant d'autres alors que le spectateur ou le lecteur cherche encore un sens à tout ce bavardage.

Chercher le sens $d u$ bavardage dans ce bavardage est cependant faire fausse route. Toute la tradition du théâtre nous a habitués à lire l'action dans les dialogues. Or, l'univers particulier de Boucher, qui avait déjà fait jouer Natures mortes en 1993 et Motel Hélène en 1997, repose sur tout autre chose. Comme les pièces précédentes, 24 poses est une réflexion sur le fait divers, sur l'événement qui survient sans prévenir, hors de la volonté des personnages. L'événement est ce qui interrompt le dialogue et non ce qui le motive. La mort est donc un instantané de la vie ordinaire, qui laisse les acteurs pantois, sans mots, avec leur douleur. Il s'agit de montrer la vie dans ce qu'elle a d'incohérent, d'inexplicable, d'indicible.

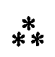

C'est un excellent indice de l'état du théâtre au Québec que de constater que l'insipide Jacynthe, de Laval ${ }^{6}$, 
de René Gingras, a été créée avec un certain succès sur la scène du Théâtre d'Aujourd'hui, le 12 janvier 2000, dans une mise en scène d'Yves Desgagnés, alors que le $D^{\prime}$ Avila $^{7}$ du même auteur, traîne toujours sur le bureau d'éventuels metteurs en scène, après avoir fait l'objet d'une lecture publique en décembre 1997 , dans le cadre de la Semaine de la dramaturgie organisée par le Centre des auteurs dramatiques et le même Théâtre d'Aujourd'hui. Gingras écrit peu et de manière inégale. On se rappellera peut-être sa première pièce Syncope qui avait obtenu le prix du gouverneur général en 1983; les amateurs ajouteront Le facteur réalité (1985). Puis quinze ans de quasi-silence, consacrés à la traduction et à l'enseignement (Gingras est titulaire de la section d'écriture dramatique à l'École Nationale de' Théâtre). Peut-on imaginer pièces aussi dissemblables que les deux qu'il publie la même année, comme pour souligner son retour? Entre l'héroüne de banlieue, qui rêve de participer à un quiz télévisé, et Thérèse, preux chevalier (chevalière?) qui fonde des carmels comme autant de moulins à vent destinés à souffler l'Inquisition, peut-il y avoir quelque chose en commun? Outre le fait qu'il s'agisse de deux femmes, que la première est désignée par son prénom puis le nom de la ville et qu'à la seconde suffit le nom de la ville, tellement celle-ci lui est identifiée, on voit mal ce qui pourrait les réunir.

Curieux texte que Jacynthe, de Laval. Une première version de la pièce avait été lue dans le cadre de la Semaine de la dramaturgie, organisée par le Centre des auteurs dramatiques, sous la direction de Martin
Faucher. La pièce était alors un monologue et elle aurait sans doute dû le rester, puisque les deux personnages masculins ne sont que des fairevaloir.

Habituée des quiz télévisés et en particulier de "La Bonne étoile", Jacynthe est prête à tout pour gagner le concours de la terrestre ordinaire, "dont le témoignage, préenregistré, voyagera aux côtés des symphonies de Mozart et d'autres équations d'Einstein, dans la première sonde interstellaire mondiale lancée expressément à l'intention d'éventuelles présences extra planétaires. Plus de cent cinquante télévisions participantes.... (p. 37) Comme il faut bien se démarquer de tous les autres candidats, Jacynthe, qui est aussi une travailleuse sociale, saisit l'occasion qui se présente à elle quand un évadé de prison, sur lequel pèse une menace d'extradition vers les États-Unis, la consulte et tente d'obtenir qu'elle intercède en sa faveur auprès des autorités policières. La pièce commence au moment où Jacynthe et Zacharie, ayant patiemment attendu la fin du quiz télévisé, prennent d'assaut la régie, simulant une prise d'otage et menaçant de tout faire sauter. L'intervention de Jean-Nil, le mari de Jacinthe, qui est aussi le rédacteur des bulletins météo de la station, tourne au guignol: "les chances que ta femme prenne d'assaut les ondes hertziennes en te mettant au défi de la convaincre de rentrer en disant "je t'aime"... sont quand même infinitésimales", dira-t-il, en résumant le propos de la pièce du même coup. On passera rapidement sur la satire de la petite-bourgeoisie banlieusarde que le titre sous-tend et, à la question existentielle que pose enfin Jacynthe: 
"est-ce que j'aurai marqué une toute, toute petite différence... quelque part?", on donnera la réponse qui s'impose: Non!

D'Avila est sous-titrée Une bistoire, avec l'adjectif indéfini. Aussi sommes-nous quelque part entre le fictif et l'historique, entre une histoire et l'Histoire. L'auteur nous met en garde: "la pièce est un amalgame de beaucoup de véracités historiques et de quelques mythifications" (p. 6). Dans l'Espagne médiévale, deux personnages se rencontrent: Thérèse, le personnage historique qui écrit pour transformer son monde et Don Quichotte, personnage de fiction en quête d'un poète qui lui donnerait vie (Ô Pirandello!). Quichotte apparaît d'abord comme un spectre à Lorenzo, le frère de Thérèse qui s'apprête à quitter D'Avila pour aller rejoindre Pizarro et porter la bonne nouvelle au Nouveau Monde, puis à Ana de San Bartolome, la jeune nonne qui assiste Thérèse dans ses œuvres. Comme son frère, "un épouvantail de chevalier qui, se croyant aimé de la plus belle des femmes, monterait pour l'impressionner à l'assaut des moulins à vent "(p. 66), Thérèse se présente comme * un chevalier errant " qui fait face à l'Inquisition en fondant des carmels.

La pièce est divisée en six tableaux que l'auteur qualifie de "gigognes ", c'est-à-dire qu'ils initient une action qui se termine au début du tableau suivant, par un récit. Chacun des tableaux porte un titre et renvoie à une œuvre, depuis Le chevalier d'Avila, somme de leurs rêves d'enfants que le frère et la sœur ont rédigée en commun, jusqu'au Libro de ma vida, l'autobiographie saisie par l'Inquisition, en passant par un poème de Jean de la Croix, lui aussi saisi par l'Inquisition: "Je suis homme et théologien comme eux. Ils ne comprendraient pas que je n'écrive pas dans leur langue." (p. 50) La pièce se termine après la mort de Thérèse, "celle dont le procès de béatification s'ouvre en un temps record" et que la famille royale proclame tout de suite "patronne d'Espagne" (p. 68). Procès de béatification qui est aussi celui de la mythification d'une femme bien réelle, dont le corps est découpé en morceaux. La pièce se clôt sur cette puissante figure d'un cadavre dispersé en autant de reliques.

Visiblement, Gingras a de la difficulté à terminer ses textes. Parti d'une idée assez intéressante dans $\mathrm{Ja}$ cynthe, de Laval, il bifurque et tourne en rond avant de boucler le tout par une pirouette somme toute assez insipide. Le caractère biographique, voire hagiographique D'Avila, impose un cadre plus strict qui permet à la pièce de retenir un peu de cohérence jusqu'à la fin. L'unité du texte est maintenue par le personnage et par la superposition de Thérèse et de Don Quichotte, dont l'esprit anime les personnages à une des époques les plus troubles de l'histoire espagnole. Serait-ce que les visions sont plus puissantes que les réalités? Quoi qu'il en soit, il me paraît que, à force de ne pas vouloir imposer de sens à ses textes, à vouloir privilégier l'image au récit, Gingras finit par rater le coche.

1. On lira, à ce sujet, le dossier que la revue Protée a consacré à - La mort de Molière et des autres ", vol. XXVII, $\mathrm{n}^{\circ} 1$, printemps 1999 , sous la direction de Rodrigue Villeneuve.

2. Robert Lalonde, Monsieur Bovary ou mourir au thêâtre, Montréal, Boréal, 2001, 227 p. 
3. Claude Paiement, L'ahurissant vertige de M. Maelström. Variations jazzées sur un thème ionesquien. Comédie, Montréal, Lanctôt éditeur, coll. - Humour -, 2001, 162 p.

4. Gilles-Philippe Pelletier, Dans la gueule d'un chien. Pièce en un acte, Montréal, Éditions Élaeis, coll. *Acta fabula *, 2000, p. 76.

5. Serge Boucher, 24 poses (portraits), Montréal, Dramaturges éditeurs, 1999, 139 p.
6. René Gingras, Jacynthe, de Laval, Carnières, Éditions Lansman, coll. *Nocturnes Théâtre : $\mathrm{n}^{\circ} 78,2000,45 \mathrm{p}$.

7. René Gingras, D'Avila. Une bistoire, Carnières, Éditions Lansman, coll. - Nocturnes Théâtre :, n 70, 1999, 70 p. 